\title{
SOLAR TRANSITION REGION AND CORONAL RESPONSE TO HEATING RATE PERTURBATIONS
}

\author{
John T. Mariska \\ E. O. Hulburt Center for Space Research \\ Naval Research Laboratory, Washington, DC
}

\section{OVERVIEW}

The solar transition region is in a dynamic state characterized by impulsively upflowing plasma and continually downflowing plasma. Using numerical simulations, I examine the conjecture that the areas of downflowing plasma are simply the base regions of coronal loops in which the heating rate is gradually decreasing and the areas of upflowing plasma are the base regions of coronal loops in which the heating rate is gradually increasing.

Only when the heating in a coronal loop is reduced to $1 \%$ of the initial value required to maintain the loop do appreciable downflow velocities develop at $10^{5} \mathrm{~K}$. The time scale over which the heating is reduced is not of great importance. Moreover, maximum downflow velocities are present only after the loop has cooled to the point where the emission measure at $10^{5} \mathrm{~K}$ is very small, making them difficult to observe.

Appreciable upflow velocities are only present in the case in which the loop that has been cooled by reducing the heating rate to $1 \%$ of the initial rate is reheated. These upflow velocities at $10^{5} \mathrm{~K}$ are much smaller than those observed at high spatial resolution in impulsive events. Larger velocities are present in the higher temperature plasma in the loop early in the heating. At this stage, however, the emission measure at high temperatures is small, making it unlikely that the plasma could be observed.

The calculations suggest that gradually reducing or increasing the heating in a magnetic flux tube will not result in plasma motions that are similar to those that are observed at high spatial resolution in the UV. A more detailed description of the calculations is presented in Mariska (1986).

\section{COOLING}

The initial model consists of a static atmosphere in a loop with a half-length of $21,000 \mathrm{~km}$. At the base of the model is a $1050 \mathrm{~km}$ thick chromospheric region in hydrostatic equilibrium at $10^{4} \mathrm{~K}$. Above this region are a transition region and corona whose properties are determined by solving the static force and energy balance equations. The base pressure here is 0.25 dynes $\mathrm{cm}^{-2}$, a value typical for the quiet sun. The background heating rate required for this initial model is $2.71 \times 10^{-4} \mathrm{ergs} \mathrm{cm}^{-3} \mathrm{~s}^{-1}$. This results in a peak temperature for the initial half loop of $1.06 \times 10^{6} \mathrm{~K}$.

Beginning with this initial model, I reduce the heating everywhere to $10 \%$ and $1 \%$ of the initial rate on time scales of 100,1000 , and $2000 \mathrm{~s}$. Figure 1 shows the evolution of the volume emission measure and the velocity at $10^{5} \mathrm{~K}$. The emission measure plot is for the calculation in which the heating was reduced to $1 \%$ of the initial rate with a time scale of 100 s. The velocity plot shows the velocity evolution for the $1 \%$ case for all three time scales.

Early in the cooling the shape of the emission measure curve remains relatively unchanged. The high-temperature turnover simple moves to lower temperatures accompanied by a gradual decrease in the slope near $10^{5} \mathrm{~K}$. As the cooling continues, the slope above $10^{5}$ 
$\mathrm{K}$ changes from positive to negative. This negative slope continues to the final quasisteady equilibrium at then end of the simulation.

As the loop cools, appreciable velocities develop at $10^{5} \mathrm{~K}$. The maximum downflow velocity for all three time scales is near $15 \mathrm{~km} \mathrm{~s}^{-1}$. Note, however, that the downflow velocity is only large for a time interval of less than $500 \mathrm{~s}$. Moreover, comparison with the emission measure plot shows that the maximum downflow velocities are present only when the emission measure has fallen to very small values at $10^{5} \mathrm{~K}$. Thus the maximum downflow velocities are present only when it is becoming increasingly difficult to observe them.

\section{HEATING}

Beginning with the atmospheric structures at the end of the cooling calculations, I increase the heating everywhere back to the initial value on time scales of 100,1000 , and 2000 s. Figure 2 shows the evolution of the volume emission measure and the velocity at $10^{5} \mathrm{~K}$. The emission measure plot is for the calculation in which the model which has been cooled by reducing the heating rate to $1 \%$ of the initial value is reheated on a $100 \mathrm{~s}$ time scale. The velocity plot shows the velocity behavior for all three time scales for the restoration of heating to the $1 \%$ model.

When the heating is restored, the temperature in the coronal portions of the loop increases very rapidly while the density remains relatively unchanged. This causes the emission measure curve to shift to the right, with some increase in absolute value. Once the mass motions have begun to redistribute plasma in the loop the slope of the emission measure also begins to change. The shape of the emission measure curve reaches its final configuration within just a few hundred seconds. For the remainder of the evolution, the loop simply fills with plasma which increases that absolute value of the emission measure at all temperatures.

The initial response of the velocity at $10^{5} \mathrm{~K}$ to the restoration of heating is a brief downflow. This is due to the sudden rise in the pressure in the coronal portions of the model pushing the entire transition region downward. The upflow velocities from evaporation for all three time scales are small and last for only a short time. Comparison of the velocity and emission measure plots shows that these upflows are largest when the emission measure at $10^{5} \mathrm{~K}$ is still small compared with its final value. Thus the velocities would be difficult to observe.

\section{DISCUSSION}

These heating and cooling calculations suggest that simple changes in the heating rate in a loop will not reproduce the mass motions observed in the quiet solar transition region. The calculations are unable to reproduce the large downflows seen at $10^{5} \mathrm{~K}$, the shape of the emission measure curve below about $10^{5} \mathrm{~K}$, or the formation of cool spicular upflows. It appears that instead some kind of steady driving mechanism, such as a heating rate asymmetry (e.g., Boris and Mariska 1982; McClymont and Craig 1986), is required.

This work was sponsored by the NASA Solar Terrestrial Theory Program and by the Office of Naval Research.

\section{REFERENCES}

Boris, J. P., and Mariska, J. T. 1982, Ap. J. (Letters), 258, L49.

Mariska, J. T. 1986, Ap. J., submitted.

McClymont, A. N., and Craig, I. J. D. 1986, Nature, 324, 128. 

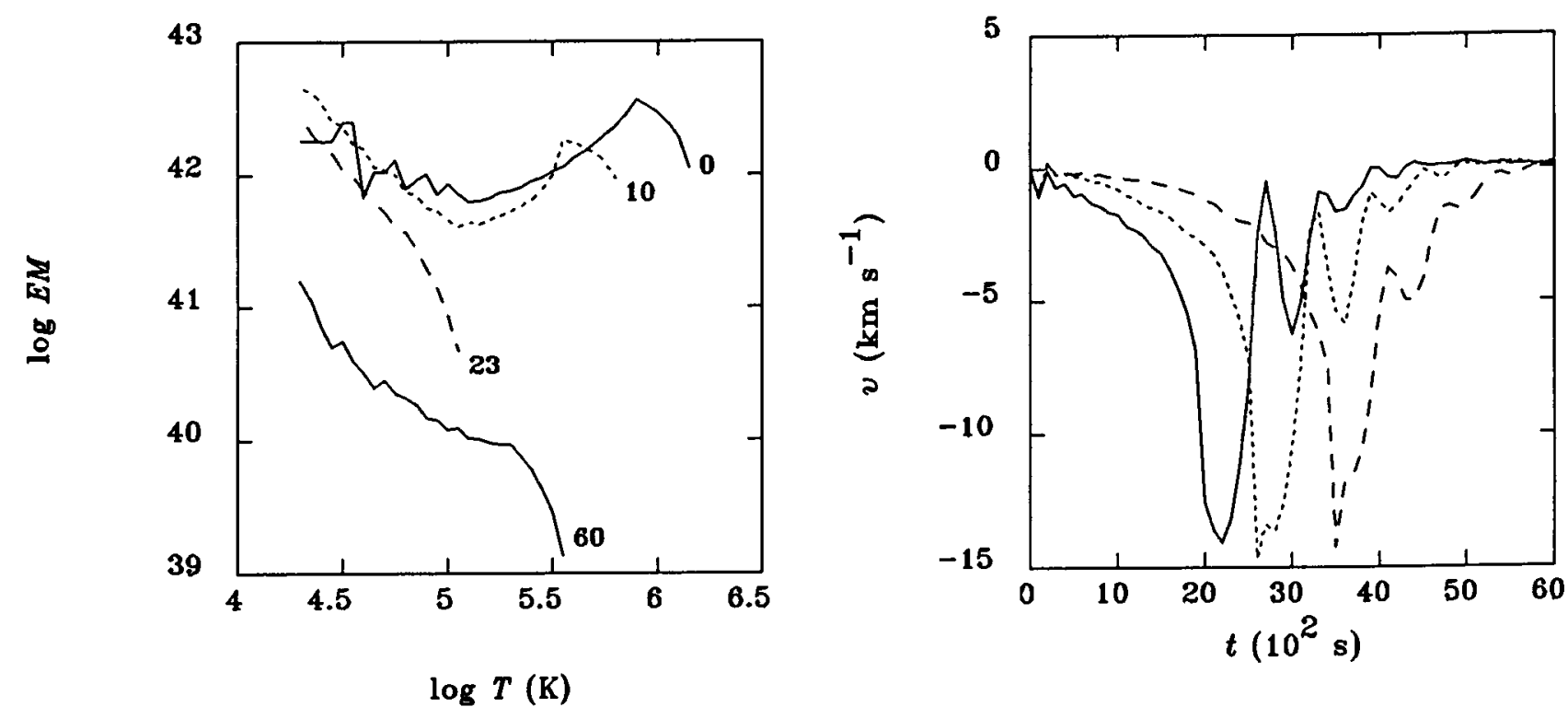

Fig. 1.-The evolution of the volume emission measure and the velocity at $10^{5} \mathrm{~K}$ for the cooling calculation in which the heating was reduced to $1 \%$ of the initial rate. The times in the emission measure plot are in units of $100 \mathrm{~s}$. In the velocity plot the solid line is for the $100 \mathrm{~s}$ time scale for the reduction in the heating, the short dashed line is for the $1000 \mathrm{~s}$ time scale, and the long dashed line is for the $2000 \mathrm{~s}$ time scale.
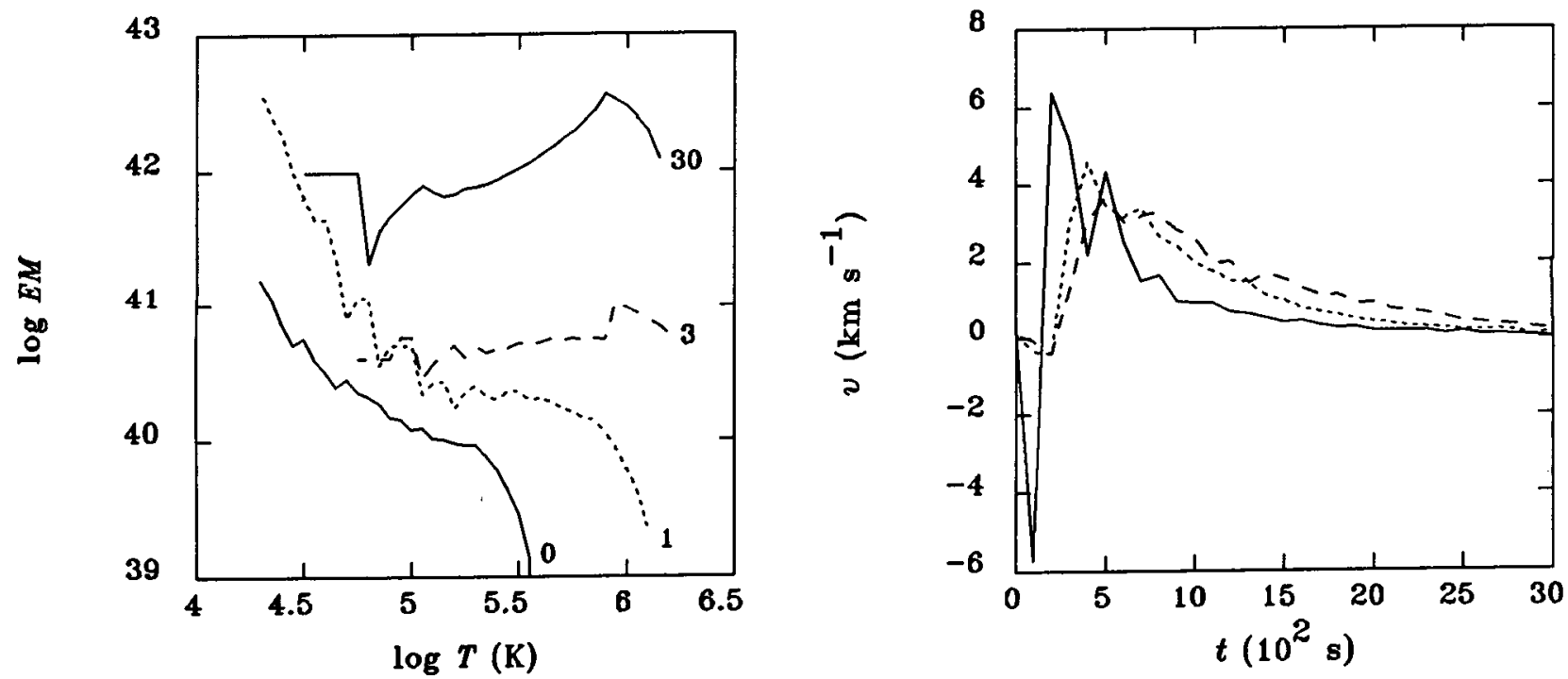

Fig. 2.-The evolution of the volume emission measure and the velocity at $10^{5} \mathrm{~K}$ for the calculation in which the heating was restored to the atmosphere that had been cooled by reducing the heating rate to $1 \%$ of the initial rate. The labeling on the plots follows the same conventions as in Figure 1. 